\title{
On level by level equivalence and inequivalence between strong compactness and supercompactness
}

\author{
by
}

\author{
Arthur W. Apter (New York, NY)
}

\begin{abstract}
We prove two theorems, one concerning level by level inequivalence between strong compactness and supercompactness, and one concerning level by level equivalence between strong compactness and supercompactness. We first show that in a universe containing a supercompact cardinal but of restricted size, it is possible to control precisely the difference between the degree of strong compactness and supercompactness that any measurable cardinal exhibits. We then show that in an unrestricted size universe containing many supercompact cardinals, it is possible to have significant failures of GCH along with level by level equivalence between strong compactness and supercompactness, except possibly at inaccessible levels.
\end{abstract}

1. Introduction and preliminaries. In [6], Shelah and the author began the study of level by level equivalence between strong compactness and supercompactness by proving the following theorem.

THEOREM 1. Let $V \vDash " Z F C+\mathfrak{K}$ is the class of supercompact cardinals". There is then a partial ordering $\mathbb{P} \subseteq V$ so that $V^{\mathbb{P}} \vDash$ "ZFC+GCH+ $\mathfrak{K}$ is the class of supercompact cardinals + For every pair of regular cardinals $\kappa<\lambda$, $\kappa$ is $\lambda$ strongly compact iff $\kappa$ is $\lambda$ supercompact, except possibly if $\kappa$ is a measurable limit of cardinals $\delta$ which are $\lambda$ supercompact".

In any model witnessing the conclusions of Theorem 1 , we will say that level by level equivalence between strong compactness and supercompactness holds. Note that the exception in Theorem 1 is provided by a theorem of

2000 Mathematics Subject Classification: 03E35, 03E55.

Key words and phrases: supercompact cardinal, strongly compact cardinal, nonreflecting stationary set of ordinals, level by level equivalence, level by level inequivalence.

The author's research which led to Theorem 2 was partially supported by PSC-CUNY Grant 61449-00-30 and by the Logic Institute of the University of Vienna. In particular, the author wishes to thank Sy Friedman, Ralf-Dieter Schindler, and their families for the hospitality shown him when he visited Vienna during his sabbatical. The author also wishes to thank the referee for helpful comments and suggestions which have been incorporated into this version of the paper. 
Menas [18], who showed that if $\kappa$ is a measurable limit of cardinals $\delta$ which are $\lambda$ strongly compact, then $\kappa$ is $\lambda$ strongly compact but need not be $\lambda$ supercompact. Observe also that Theorem 1 is a strengthening of the result of Kimchi and Magidor [15], who showed it is consistent for the classes of strongly compact and supercompact cardinals to coincide precisely, except at measurable limit points.

The purpose of this paper is to continue the investigation begun in [6] by establishing two new theorems, one concerning level by level inequivalence between strong compactness and supercompactness, and one concerning level by level equivalence between strong compactness and supercompactness in the context of the failure of GCH. Specifically, we prove the following, taking as a notational convention for the rest of the paper that for $\delta$ any non-supercompact measurable cardinal, $\theta_{\delta}$ is the least cardinal so that $\delta$ is not $\theta_{\delta}$ supercompact, and for $\alpha$ an arbitrary ordinal, $\lambda_{\alpha}$ is the least inaccessible cardinal above $\alpha$.

THEOREM 2. Let $V \vDash " Z F C+G C H+\kappa_{0}$ is $\varrho=\lambda_{\kappa_{0}}$ supercompact + There is some supercompact ultrafilter $\mathcal{U}$ over $P_{\kappa_{0}}(\varrho)$ so that for $j: V \rightarrow M$ with $j=j_{\mathcal{U}}$ the associated elementary embedding, $M \vDash$ " $\kappa_{0}$ is @ supercompact" ". There are then cardinals $\kappa, \lambda<\kappa_{0}$ and a partial ordering $\mathbb{P} \in V$ so that for $V^{*}$ the model $V^{\mathbb{P}}$ truncated at $\lambda, V^{*} \vDash " Z F C+G C H+\kappa$ is supercompact + No cardinal $\delta>\kappa$ is inaccessible + If $\delta<\kappa$ is measurable, then $\delta$ is $\theta_{\delta}^{+5}$ strongly compact but is not $\theta_{\delta}^{+6}$ strongly compact".

TheOREM 3. Let $V \vDash " Z F C+\mathfrak{K} \neq \emptyset$ is the class of supercompact cardinals". There is then a partial ordering $\mathbb{P} \subseteq V$ so that $V^{\mathbb{P}} \vDash " Z F C+\mathfrak{K}$ is the class of supercompact cardinals $+2^{\delta}=\delta^{++}$if $\delta$ is inaccessible $+2^{\delta}=\delta^{+}$ if $\delta$ is not inaccessible + For every pair of regular cardinals $\kappa<\lambda, \kappa$ is $\lambda$ strongly compact iff $\kappa$ is $\lambda$ supercompact, except possibly if $\kappa$ is a measurable limit of cardinals $\delta$ which are $\lambda$ supercompact, or $\lambda$ is inaccessible".

We take this opportunity to make several comments concerning Theorems 2 and 3 . We note that in Theorem 2, we have produced a model containing a supercompact cardinal $\kappa$ with no inaccessibles above it so that the degree of level by level inequivalence between strong compactness and supercompactness below $\kappa$ is precisely controlled, in the sense we know that for any measurable cardinal $\delta, \delta$ 's degree of supercompactness fails right at $\theta_{\delta}$, yet $\delta$ must be exactly $\theta_{\delta}^{+5}$ strongly compact. Also, as our proof will indicate, there is nothing special about +5 indicating the degree of level by level inequivalence below $\kappa$ between strong compactness and supercompactness. It will be possible, e.g., for the degree of level by level inequivalence between strong compactness and supercompactness for $\delta$ to be given by $+\varphi(\delta)$ (meaning that $\delta$ is $\theta_{\delta}^{+\varphi(\delta)}$ strongly compact but $\delta$ is not $\theta_{\delta}^{+\varphi(\delta)+1}$ 
strongly compact), where $\varphi(x)$ is a formula in one free variable that defines a function sending any ordinal $\alpha$ to an ordinal strictly below the least inaccessible above $\alpha$, subject to one restriction. This is that $\theta_{\delta}^{+\varphi(\delta)}$ cannot be a singular (strong limit) cardinal of cofinality below $\delta$. We will comment on this in greater detail immediately following the proof of Theorem 2. Finally, Theorem 3 should be contrasted with Theorem 4 of [2], where a model containing a supercompact cardinal $\kappa$ with no measurables above $\kappa$ and in which no cardinal $\delta$ is $\lambda$ supercompact for $\lambda>\delta$ measurable is produced in which there is precise level by level equivalence below $\kappa$ between strong compactness and supercompactness, yet for every measurable cardinal $\delta, \delta$ is $\delta^{+}$supercompact and $2^{\delta}=\delta^{++}$. In Theorem 3 of this paper, there are no restrictions on the size of the universe, yet there remains the ambiguity of whether $\kappa$ is $\lambda$ strongly compact iff $\kappa$ is $\lambda$ supercompact, whenever $\lambda$ is inaccessible and $\kappa$ is not a measurable limit of cardinals $\delta$ which are $\lambda$ supercompact.

Before giving the proofs of our theorems, we briefly mention some preliminary information. Essentially, our notation and terminology are standard, and when this is not the case, this will be clearly noted. For $\alpha<\beta$ ordinals, $[\alpha, \beta],[\alpha, \beta),(\alpha, \beta]$, and $(\alpha, \beta)$ are as in standard interval notation.

When forcing, $q \geq p$ will mean that $q$ is stronger than $p$. If $G$ is $V$-generic over $\mathbb{P}$, we will use both $V[G]$ and $V^{\mathbb{P}}$ to indicate the universe obtained by forcing with $\mathbb{P}$. If $x \in V[G]$, then $\dot{x}$ will be a term in $V$ for $x$. If also $\kappa$ is inaccessible and $\mathbb{P}=\left\langle\left\langle\mathbb{P}_{\alpha}, \dot{\mathbb{Q}}_{\alpha}\right\rangle: \alpha<\kappa\right\rangle$ is an Easton support iteration of length $\kappa$ so that at stage $\alpha$, a non-trivial forcing is done adding a subset of $\delta_{\alpha}$, then we will say that $\delta_{\alpha}$ is in the field of $\mathbb{P}$. We may, from time to time, confuse terms with the sets they denote and write $x$ when we actually mean $\dot{x}$, especially when $x$ is some variant of the generic set $G$, or $x$ is in the ground model $V$.

Let $\kappa$ be a regular cardinal. The partial ordering $\mathbb{P}$ is $\kappa$-directed closed if for every cardinal $\delta<\kappa$ and every directed set $\left\langle p_{\alpha}: \alpha<\delta\right\rangle$ of elements of $\mathbb{P}$ (where $\left\langle p_{\alpha}: \alpha<\delta\right\rangle$ is directed if for any two distinct elements $p_{\varrho}, p_{\nu} \in$ $\left\langle p_{\alpha}: \alpha<\delta\right\rangle, p_{\varrho}$ and $p_{\nu}$ have a common upper bound of the form $\left.p_{\sigma}\right)$ there is an upper bound $p \in \mathbb{P}$. $\mathbb{P}$ is $\kappa$-strategically closed if in the two-person game in which the players construct an increasing sequence $\left\langle p_{\alpha}: \alpha \leq \kappa\right\rangle$, where player I plays odd stages and player II plays even and limit stages (choosing the trivial condition at stage 0), player II has a strategy which ensures the game can always be continued. Note that if $\mathbb{P}$ is $\kappa$-strategically closed and $f: \kappa \rightarrow V$ is a function in $V^{\mathbb{P}}$, then $f \in V . \mathbb{P}$ is $\prec \kappa$-strategically closed if in the two-person game in which the players construct an increasing sequence $\left\langle p_{\alpha}: \alpha<\kappa\right\rangle$, where player I plays odd stages and player II plays even and limit stages, player II has a strategy which ensures the game can always be continued. 
Suppose now that $\kappa<\lambda$ are regular cardinals. A partial ordering $\mathbb{P}(\kappa, \lambda)$ that will be used in the proof of Theorem 2 is the partial ordering for adding a non-reflecting stationary set of ordinals of cofinality $\kappa$ to $\lambda$. Specifically, $\mathbb{P}(\kappa, \lambda)=\{p:$ For some $\alpha<\lambda, p: \alpha \rightarrow\{0,1\}$ is the characteristic function of $S_{p}$, a subset of $\alpha$ not stationary at its supremum nor having any initial segment which is stationary at its supremum, so that $\beta \in S_{p}$ implies $\beta>\kappa$ and $\operatorname{cof}(\beta)=\kappa\}$, ordered by $q \geq p$ iff $q \supseteq p$ and $S_{p}=S_{q} \cap \sup \left(S_{p}\right)$, i.e., $S_{q}$ is an end extension of $S_{p}$. It is well-known that for $G V$-generic over $\mathbb{P}(\kappa, \lambda)$ (see [7], [3], or [15]), in $V[G]$, if we assume GCH holds in $V$, a non-reflecting stationary set $S=S[G]=\bigcup\left\{S_{p}: p \in G\right\} \subseteq \lambda$ of ordinals of cofinality $\kappa$ has been introduced, the bounded subsets of $\lambda$ are the same as those in $V$, and cardinals, cofinalities, and GCH have been preserved. It is also virtually immediate that $\mathbb{P}(\kappa, \lambda)$ is $\kappa$-directed closed, and it can be shown (see [7], [3], or [15]) that $\mathbb{P}(\kappa, \lambda)$ is $\prec \lambda$-strategically closed.

In the proof of Theorem 3 , we will employ the standard Cohen partial ordering $\operatorname{Add}(\kappa, \lambda)$ for adding $\lambda$ subsets of $\kappa$. As opposed to the most common usage, however, where $\kappa<\lambda$ are both regular cardinals, we will allow $\lambda$ to be an arbitrary ordinal when appropriate. Assuming GCH holds for cardinals at and above $\kappa$, this will not change the fact that $\operatorname{Add}(\kappa, \lambda)$ is $\kappa$-directed closed and $\kappa^{+}$-c.c.

We mention that we are assuming familiarity with the large cardinal notions of measurability, strong compactness, and supercompactness. Interested readers may consult [14] or [19] for further details. We only note that the cardinal $\kappa$ is $<\lambda$ supercompact if $\kappa$ is $\delta$ supercompact for every cardinal $\delta<\lambda$.

2. The proof of Theorem 2. Let $V$ be a model for the hypotheses of Theorem 2, i.e., assume $V \vDash$ "ZFC $+\mathrm{GCH}+\kappa_{0}$ is $\varrho=\lambda_{\kappa_{0}}$ supercompact + There is some supercompact ultrafilter $\mathcal{U}$ over $P_{\kappa_{0}}(\varrho)$ so that for $j$ : $V \rightarrow M$ with $j=j_{\mathcal{U}}$ the associated elementary embedding, $M \vDash " \kappa_{0}$ is $\varrho$ supercompact" ". This allows us to define the first partial ordering $\mathbb{P}^{0}$ we use in the proof of Theorem 2 as the Easton support iteration of length $\kappa_{0}$ which begins by adding a Cohen subset of $\omega$ and then adds, to every measurable cardinal $\delta<\kappa_{0}$ which is not $\lambda_{\delta}$ supercompact, a non-reflecting stationary set of ordinals of cofinality $\omega$. Since $M \vDash$ " $\kappa_{0}$ is $\varrho$ supercompact and $\varrho$ is the least inaccessible cardinal above $\kappa_{0}$ ", by reflection, $A=\left\{\delta<\kappa_{0}: \delta\right.$ is $\lambda_{\delta}$ supercompact $\}$ is unbounded in $\kappa_{0}$.

We now sketch the proof of Lemma 3.2 of [3], which allows us to infer that $V^{\mathbb{P}^{0}} \vDash$ "For every cardinal $\delta<\kappa_{0}, \delta$ is measurable iff $\delta$ is $\lambda_{\delta}$ strongly compact". By Hamkins' work of [9]-[11] and the fact that every element of $\kappa_{0}-A$ contains a non-reflecting stationary set of ordinals of cofinality $\omega$, $V^{\mathbb{P}^{0}} \vDash$ "The only measurable cardinals $\delta<\kappa_{0}$ are those $\delta$ which were $\lambda_{\delta}$ 
supercompact in $V "$. For any $\delta<\kappa_{0}$ which is $\lambda_{\delta}$ supercompact in $V$, write $\mathbb{P}^{0}=\mathbb{P}_{\delta} * \dot{\mathbb{P}}^{\delta}$, where the field of $\mathbb{P}_{\delta}$ is composed of ordinals below $\delta$. From this factorization, we see that $\vdash_{\mathbb{P}_{\delta}}$ " $\dot{\mathbb{P}}^{\delta}$ is $2^{\lambda_{\delta}}$-strategically closed". Thus, it is the case that $V^{\mathbb{P}^{0}} \vDash$ " $\delta$ is $\lambda_{\delta}$ strongly compact" iff $V^{\mathbb{P}_{\delta}} \vDash$ " $\delta$ is $\lambda_{\delta}$ strongly compact". The fact that $V^{\mathbb{P}_{\delta}} \vDash$ " $\delta$ is $\lambda_{\delta}$ strongly compact", however, follows using an argument due to Magidor, unpublished by him but exposited in [3]-[5], and, in a more complicated form, in Claim 1 of Case 2 of Lemma 2.3 of this paper. This completes our proof sketch of Lemma 3.2 of [3]. In addition, the usual Easton arguments show $V^{\mathbb{P}^{0}} \vDash \mathrm{GCH}$, and since $\mathbb{P}^{0}$ can be defined so that $\left|\mathbb{P}^{0}\right|=\kappa_{0}, V^{\mathbb{P}^{0}} \vDash$ " $\varrho$ is the least inaccessible cardinal above $\kappa_{0} "$.

\section{LeMma 2.1. $V^{\mathbb{P}^{0}} \vDash$ " $\kappa_{0}$ is @ supercompact".}

Proof. Let $H_{0}$ be $V$-generic over $\mathbb{P}^{0}$, and let $j: V \rightarrow M$ be as in the hypotheses of Theorem 2. Since $M \vDash$ " $\varrho$ is the least inaccessible cardinal above $\kappa_{0}$ and $\kappa_{0}$ is $\varrho$ supercompact", $j\left(\mathbb{P}^{0}\right)=\mathbb{P}^{0} * \dot{\mathbb{Q}}$, where $\kappa_{0} \notin$ field $(\dot{\mathbb{Q}})$ and the least ordinal in the field of $\dot{\mathbb{Q}}$ is above $\varrho$. Therefore, as $M\left[H_{0}\right] \vDash "|\mathbb{Q}|=j\left(\kappa_{0}\right)$ and $\left|2^{\mathbb{Q}}\right|=j\left(\kappa_{0}\right)^{+}=j\left(\kappa_{0}^{+}\right) "$ and $V \vDash "\left|j\left(\kappa_{0}^{+}\right)\right|=\left|\left\{f: f: P_{\kappa_{0}}(\varrho) \rightarrow \kappa_{0}^{+}\right\}\right|=$ $\left|\left[\kappa_{0}^{+}\right]^{\varrho}\right|=\varrho^{+} ", V\left[H_{0}\right] \vDash$ "There are $\varrho^{+}$subsets of $\mathbb{Q}$ present in $M\left[H_{0}\right]$ ". Hence, we can let $\left\langle D_{\alpha}: \alpha<\varrho^{+}\right\rangle$enumerate in $V\left[H_{0}\right]$ all dense open subsets of $\mathbb{Q}$ present in $M\left[H_{0}\right]$, where for the purposes of the argument to be given below, we assume without loss of generality that for every dense open set $D \in\left\langle D_{\alpha}: \alpha<\varrho^{+}\right\rangle, D=D_{\beta}$ for some odd ordinal $\beta$.

By the fact $M^{\varrho} \subseteq M, \Vdash_{\mathbb{P}^{0}}$ "⿺辶் is $\prec \varrho^{+}$-strategically closed" in both $M$ and $V$. Therefore, since standard arguments show $M\left[H_{0}\right]$ remains $\varrho$ closed with respect to $V\left[H_{0}\right], V\left[H_{0}\right] \vDash$ "Q⿱Q is $\prec \varrho^{+}$-strategically closed". We can now construct an $M\left[H_{0}\right]$-generic object $H$ over $\mathbb{Q}$ in $V\left[H_{0}\right]$ as follows. Players I and II play a game of length $\varrho^{+}$. The initial pair of moves is generated by player II choosing the trivial condition $q_{0}$ and player I responding by choosing $q_{1} \in D_{1}$. Then, at an even stage $\alpha+2$, player II picks $q_{\alpha+2} \geq q_{\alpha+1}$ by using some fixed strategy $\mathcal{S}$, where $q_{\alpha+1}$ was chosen by player I to be so that $q_{\alpha+1} \in D_{\alpha+1}$ and $q_{\alpha+1} \geq q_{\alpha}$. If $\alpha$ is a limit ordinal, player II uses $\mathcal{S}$ to pick $q_{\alpha}$ extending each $q_{\beta}$ for $\beta<\alpha$. By the $\prec \varrho^{+}$-strategic closure of $\mathbb{Q}$ in both $M\left[H_{0}\right]$ and $V\left[H_{0}\right]$, the sequence $\left\langle q_{\alpha}: \alpha<\varrho^{+}\right\rangle$as just described exists. By construction, $H=\left\{p \in \mathbb{Q}: \exists \alpha<\varrho^{+}\left[q_{\alpha} \geq p\right]\right\}$ is our $M\left[H_{0}\right]$-generic object over $\mathbb{Q}$. Thus, $j: V \rightarrow M$ extends in $V\left[H_{0}\right]$ to $j: V\left[H_{0}\right] \rightarrow M\left[H_{0}\right][H]$, so $V\left[H_{0}\right] \vDash$ " $\kappa_{0}$ is $\varrho$ supercompact". This completes the proof of Lemma 2.1 .

We remark that the proof of Lemma 2.1 remains valid under different circumstances. Specifically, suppose we are forcing over a ground model $V^{\prime}$ satisfying GCH. Suppose further $\kappa^{\prime}<\lambda^{\prime}$ are so that $\kappa^{\prime}$ is $\lambda^{\prime}$ supercompact, 
$\lambda^{\prime}$ is a cardinal having cofinality at least $\kappa^{\prime}, B \subseteq \kappa^{\prime}$ is an unbounded set of regular cardinals with $\omega \notin B$, and for some $j^{\prime}: V^{\prime} \rightarrow M^{\prime}$ witnessing the $\lambda^{\prime}$ supercompactness of $\kappa^{\prime}$ generated by a supercompact ultrafilter $\mathcal{U}^{\prime}$ over $P_{\kappa^{\prime}}\left(\lambda^{\prime}\right), j^{\prime}(B) \cap\left[\kappa^{\prime}, \lambda^{\prime}\right]=\emptyset$. If $\mathbb{Q}$ is an Easton support iteration which begins by adding a Cohen subset of $\omega$ and then adds, for every $\delta \in B$, a non-reflecting stationary set of ordinals of cofinality $\omega$, the proof just given shows that after forcing with $\mathbb{Q}, \kappa^{\prime}$ remains $\lambda^{\prime}$ supercompact.

Take now $V_{0}=V^{\mathbb{P}^{0}}$. Let $\kappa$ be minimal so that $V_{0} \vDash$ " $\kappa$ is $<\lambda_{\kappa}$ supercompact", and let $\lambda=\lambda_{\kappa}$. Since $V_{0} \vDash " \kappa_{0}$ is $\varrho=\lambda_{\kappa_{0}}$ supercompact", again by reflection, $\kappa, \lambda<\kappa_{0}$.

Working in $V_{0}$, let $\left\langle\delta_{\alpha}: \alpha<\kappa\right\rangle$ be an increasing enumeration of the measurable cardinals below $\kappa$. By the choice of $\kappa$ and $\lambda$, it must be the case that $\theta_{\delta}$ exists for every measurable cardinal $\delta<\kappa$ and $\theta_{\delta}<\lambda_{\delta}$. We therefore define the second partial ordering $\mathbb{P}^{1}$ we use in the proof of Theorem 2 as the Easton support iteration of length $\kappa$ which begins by adding a Cohen subset of $\omega$ and then adds, for every $V_{0}$-measurable cardinal $\delta<\kappa$, a non-reflecting stationary set of ordinals of cofinality $\omega$ to $\left(\theta_{\delta}^{+6}\right)^{V_{0}}$. Let $\mathbb{P}=\mathbb{P}^{0} * \dot{\mathbb{P}}^{1}$. Once again, the usual Easton arguments show $V_{0}^{\mathbb{P}^{1}}=V^{\mathbb{P}^{0} * \dot{\mathbb{P}}^{1}}=V^{\mathbb{P}} \vDash \mathrm{GCH}$. Also, as before, since $\mathbb{P}^{1}$ can be defined in $V_{0}$ so as to have cardinality $\kappa$, $V_{0}^{\mathbb{P}^{1}}=V^{\mathbb{P}^{0} * \dot{\mathbb{P}}^{1}}=V^{\mathbb{P}} \vDash " \lambda$ is the least inaccessible cardinal above $\kappa$ ".

LEMma 2.2. $V_{0}^{\mathbb{P}^{1}}=V^{\mathbb{P}} \vDash$ " $\kappa$ is $<\lambda$ supercompact".

Proof. Let $\delta<\lambda$ be a successor cardinal, and let $k: V_{0} \rightarrow M^{*}$ be an elementary embedding generated by a supercompact ultrafilter over $P_{\kappa}(\delta)$ so that $M^{*} \vDash$ " $\kappa$ is not $\delta$ supercompact". By the definition of $\mathbb{P}^{1}$ and the choice of $k, k\left(\mathbb{P}^{1}\right)=\mathbb{P}^{1} * \dot{\mathbb{Q}}$, where the least ordinal in the field of $\dot{\mathbb{Q}}$ is above $\left(\delta^{+}\right)^{M^{*}}=\left(\delta^{+}\right)^{V_{0}}$. Since $V_{0} \vDash \mathrm{GCH}$, this means we can apply the argument given in Lemma 2.1 to show that $V_{0}^{\mathbb{P}^{1}} \vDash " \kappa$ is $\delta$ supercompact". This completes the proof of Lemma 2.2.

LEMma 2.3. $V^{\mathbb{P}} \vDash$ "For every measurable cardinal $\delta<\kappa, \delta$ is $\theta_{\delta}^{+5}$ strongly compact but is not $\theta_{\delta}^{+6}$ strongly compact".

Proof. Let $\delta<\kappa$ be measurable in $V^{\mathbb{P}}$. Write $\mathbb{P}^{1}=\mathbb{P}^{\prime} * \dot{\mathbb{P}}^{\prime \prime}$, where $\left|\mathbb{P}^{\prime}\right|=\omega$ and $\Vdash_{\mathbb{P}^{\prime}}$ " $\dot{\mathbb{P}}$ " is $\aleph_{1}$-strategically closed". In Hamkins' terminology of [9]-[11] $\mathbb{P}^{1}$ "admits a low gap", so by the results of [9]-[11], $V_{0} \vDash$ " $\delta$ is measurable".

For $n=0,1$, write $\mathbb{P}^{n}=\mathbb{P}_{\delta}^{n} * \dot{\mathbb{P}}^{n, \delta}$, where $\mathbb{P}_{\delta}^{n}$ is the portion of $\mathbb{P}^{n}$ whose field consists of ordinals below $\delta$. We consider the following two cases.

CASE 1: $V_{0} \vDash "\left|\mathbb{P}_{\delta}^{1}\right|<\delta$ ". Since by construction, $V_{0} \vDash " \delta$ is $\lambda_{\delta}$ strongly compact", the Lévy-Solovay results [16] imply that $V_{0}^{\mathbb{P}_{\delta}^{1}} \vDash " \delta$ is $\lambda_{\delta}$ strongly compact and $\theta_{\delta}=\left(\theta_{\delta}\right)^{V_{0}}$ ". As by its definition, $\Vdash_{\mathbb{P}_{\delta}^{\frac{1}{\delta}}}$ "P $\mathbb{P}^{1, \delta}$ is $\theta_{\delta}^{+5}$-strategically closed and adds a non-reflecting stationary set of ordinals of cofinality $\omega$ to 
$\theta_{\delta}^{+6}$ ", the closure properties of $\mathbb{P}^{1, \delta}$ together with an application of Theorem 4.8 of $[19]$ and the succeeding remarks yield $V_{0}^{\mathbb{P}_{\delta}^{1} * \dot{\mathbb{P}}^{1, \delta}}=V_{0}^{\mathbb{P}^{1}}=V^{\mathbb{P}} \vDash " \delta$ is $<\theta_{\delta}$ supercompact, $\delta$ is $\theta_{\delta}^{+5}$ strongly compact, and $\delta$ is not $\theta_{\delta}^{+6}$ strongly compact".

CASE 2: $V_{0} \vDash "\left|\mathbb{P}_{\delta}^{1}\right|=\delta$ ". Let $\mathbb{P}^{*}=\mathbb{P}_{\delta}^{0} * \dot{\mathbb{P}}_{\delta}^{1}$. Note that by the definition of $\mathbb{P}$, with a slight abuse of notation, the definition of $\mathbb{P}^{*}$ just given makes sense. We prove Case 2 via a series of claims, with Claim 1 stating that $\delta$ is $\lambda_{\delta}$ strongly compact after forcing over $V$ with $\mathbb{P}^{*}$, Claim 2 stating that $\theta_{\delta}$ does not change going from $V_{0}$ to $V_{0}^{\mathbb{P}^{1}}=V^{\mathbb{P}}$, and Claim 3 stating that Claims 1 and 2 allow us to finish as in Case 1.

Claim 1. $V^{\mathbb{P}^{*}}=V^{\mathbb{P}_{\delta}^{0} * \dot{\mathbb{P}}_{\delta}^{1}} \vDash$ " $\delta$ is $\lambda_{\delta}$ strongly compact".

Proof. To prove Claim 1, we use a modification of the argument due to Magidor alluded to in the paragraph immediately preceding Lemma 2.1. By the definition of $\mathbb{P}^{0}$, it must be the case that $V \vDash$ " $\delta$ is $\lambda_{\delta}$ supercompact". Further, the definitions of $\mathbb{P}^{0}$ and $\mathbb{P}^{1}$ show that $\lambda_{\delta}$ has the same meaning in $V, V^{\mathbb{P}^{0}}, V^{\mathbb{P}^{0} * \dot{\mathbb{P}}^{1}}=V^{\mathbb{P}}$, and $V^{\mathbb{P}^{*}}$. Therefore, there is no ambiguity in choosing $k_{1}: V \rightarrow M^{* *}$ to be an elementary embedding witnessing the $\lambda_{\delta}$ supercompactness of $\delta$ generated by a supercompact ultrafilter over $P_{\delta}\left(\lambda_{\delta}\right)$ so that $M^{* *} \vDash$ " $\delta$ is not $\lambda_{\delta}$ supercompact". Since $M^{* *} \vDash$ " $\delta$ is measurable", we may choose a normal ultrafilter $\mathcal{U}^{\prime \prime}$ of Mitchell order 0 over $\delta$ so that $k_{2}: M^{* *} \rightarrow$ $N$ is an elementary embedding witnessing the measurability of $\delta$ definable in $M^{* *}$ with $N \vDash$ " $\delta$ is not measurable". It is the case that if $k: V \rightarrow N$ is an elementary embedding with critical point $\delta$ and for any $x \subseteq N$ with $|x| \leq \lambda_{\delta}$, there is some $y \in N$ so that $x \subseteq y$ and $N \vDash "|y|<k(\delta)$ ", then $k$ witnesses the $\lambda_{\delta}$ strong compactness of $\delta$. By using this fact, it is easily verifiable that $j^{*}=k_{2} \circ k_{1}$ is an elementary embedding witnessing the $\lambda_{\delta}$ strong compactness of $\delta$. We show that $j^{*}$ extends to $j^{*}: V^{\mathbb{P}^{*}} \rightarrow N^{j^{*}\left(\mathbb{P}^{*}\right)}$. This extended embedding will witness the $\lambda_{\delta}$ strong compactness of $\delta$ in $V^{\mathbb{P}^{*}}$.

To do this, write $j^{*}\left(\mathbb{P}^{*}\right)$ as $\left(\mathbb{P}_{\delta}^{0} * \dot{\mathbb{Q}}^{0} * \dot{\mathbb{R}}^{0}\right) *\left(\dot{\mathbb{P}}_{\delta}^{1} * \dot{\mathbb{Q}}^{1} * \dot{\mathbb{R}}^{1}\right)$, where for $n=0,1$, the $\dot{\mathbb{Q}}^{n}$ are terms for the portions of $j^{*}\left(\mathbb{P}^{*}\right)$ between $\delta$ and $k_{2}(\delta)$ and the $\dot{\mathbb{R}}^{n}$ are terms for the rest of $j^{*}\left(\mathbb{P}^{*}\right)$, i.e., the parts above $k_{2}(\delta)$. Note that by the definition of $\mathbb{P}_{\delta}^{0}$, since $N \vDash$ " $\delta$ is inaccessible but is not measurable", $\delta \notin$ field $\left(\dot{\mathbb{Q}}^{0}\right)$. Thus, the field of $\dot{\mathbb{Q}}^{0}$ is composed of an unbounded subset of $N$-measurable cardinals in the interval $\left(\delta, k_{2}(\delta)\right]$. As $M^{* *} \vDash " ~ \delta$ is measurable but is not $\lambda_{\delta}$ supercompact", by the definition of $\mathbb{P}_{\delta}^{0}, \delta \in \operatorname{field}\left(k_{1}\left(\mathbb{P}_{\delta}^{0}\right)\right)$. This means that by elementarity, $k_{2}(\delta) \in \operatorname{field}\left(\dot{\mathbb{Q}}^{0}\right)$. Further, the field of $\dot{\mathbb{R}}^{0}$ is also composed of an unbounded subset of the $N$-measurable cardinals in the interval $\left(k_{2}(\delta), k_{2}\left(k_{1}(\delta)\right)\right)$.

Let $H=H_{0} * H_{1}$ be $V$-generic over $\mathbb{P}^{*}=\mathbb{P}_{\delta}^{0} * \dot{\mathbb{P}}_{\delta}^{1}$. We construct in $V\left[H_{0}\right]$ an $N\left[H_{0}\right]$-generic object $H_{1}^{0}$ over $\mathbb{Q}^{0}$ and an $N\left[H_{0}\right]\left[H_{1}^{0}\right]$-generic object $H_{2}^{0}$ over 
$\mathbb{R}^{0}$. We then construct in $V\left[H_{0}\right]\left[H_{1}\right]=V[H]$ an $N\left[H_{0}\right]\left[H_{1}^{0}\right]\left[H_{2}^{0}\right]\left[H_{1}\right]$-generic object $H_{1}^{1}$ over $\mathbb{Q}^{1}$ and an $N\left[H_{0}\right]\left[H_{1}^{0}\right]\left[H_{2}^{0}\right]\left[H_{1}\right]\left[H_{1}^{1}\right]$-generic object $H_{2}^{1}$ over $\mathbb{R}^{1}$. Our construction will guarantee that $j^{* \prime \prime}\left(H_{0} * H_{1}\right) \subseteq\left(H_{0} * H_{1}^{0} * H_{2}^{0}\right) *$ $\left(H_{1} * H_{1}^{1} * H_{2}^{1}\right)$. This means that $j^{*}: V \rightarrow N$ extends to $j^{*}: V\left[H_{0} * H_{1}\right] \rightarrow$ $N\left[H_{0}\right]\left[H_{1}^{0}\right]\left[H_{2}^{0}\right]\left[H_{1}\right]\left[H_{1}^{1}\right]\left[H_{2}^{1}\right]$ in $V[H]=V\left[H_{0}\right]\left[H_{1}\right]$, meaning that $V^{\mathbb{P}^{*}} \vDash " \delta$ is $\lambda_{\delta}$ strongly compact".

To obtain $H_{1}^{0}$, note that since $k_{2}$ is generated by an ultrafilter over $\delta$ and since GCH holds in both $V$ and $N,\left|k_{2}\left(\delta^{+}\right)\right|=\left|k_{2}\left(2^{\delta}\right)\right|=\mid\left\{f: f: \delta \rightarrow \delta^{+}\right.$is a function $\}|=|\left[\delta^{+}\right]^{\delta} \mid=\delta^{+}$. Thus, as $N\left[H_{0}\right] \vDash "\left|\wp\left(\mathbb{Q}^{0}\right)\right|=k_{2}\left(2^{\delta}\right)$ ", we can let $\left\langle D_{\alpha}: \alpha<\delta^{+}\right\rangle$enumerate in either $V\left[H_{0}\right]$ or $M^{* *}\left[H_{0}\right]$ the dense open subsets of $\mathbb{Q}^{0}$ found in $N\left[H_{0}\right]$ so that for every dense open set $D \in\left\langle D_{\alpha}: \alpha<\delta^{+}\right\rangle$, $D=D_{\beta}$ for some odd ordinal $\beta$. Since $N\left[H_{0}\right] \vDash " \mathbb{Q}^{0}$ is $\prec \delta^{+}$-strategically closed", the argument given in the proof of Lemma 2.1 for the construction of the generic object $H$ is applicable here as well and allows us to build $H_{1}^{0}$ in either $V\left[H_{0}\right]$ or $M^{* *}\left[H_{0}\right]$ in the same manner.

We next construct in $V\left[H_{0}\right]$ the desired $N\left[H_{0}\right]\left[H_{1}^{0}\right]$-generic object $H_{2}^{0}$ over $\mathbb{R}^{0}$. To do this, we first note that as $\delta \in \operatorname{field}\left(k_{1}\left(\mathbb{P}_{\delta}^{0}\right)\right)$, we can write $k_{1}\left(\mathbb{P}_{\delta}^{0}\right)$ as $\mathbb{P}_{\delta}^{0} * \dot{\mathbb{S}}^{0} * \dot{\mathbb{T}}^{0}$, where $\Vdash_{\mathbb{P}_{\delta}^{0}}$ " $\dot{\mathbb{S}}^{0}=\dot{\mathbb{P}}(\omega, \delta)$ ", and $\dot{\mathbb{T}}^{0}$ is a term for the rest of $k_{1}\left(\mathbb{P}_{\delta}^{0}\right)$.

Note now that $M^{* *} \vDash$ "There are no measurable cardinals in the interval $\left(\delta, \lambda_{\delta}\right]$ ". Thus, the field of $\dot{T}^{0}$ is composed of an unbounded subset of the $M^{* *}$-measurable cardinals in the interval $\left(\lambda_{\delta}, k_{1}(\delta)\right)$, which implies that in

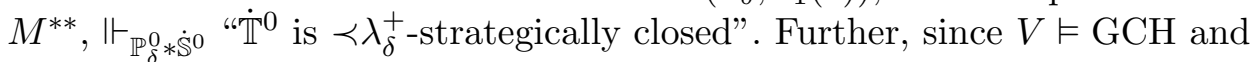
$\lambda_{\delta}$ has cofinality at least $\delta,\left|\left[\lambda_{\delta}\right]^{<\delta}\right|=\lambda_{\delta}$ and $2^{\lambda_{\delta}}=\lambda_{\delta}^{+}$. Therefore, as $k_{1}$ is generated by a supercompact ultrafilter over $P_{\delta}\left(\lambda_{\delta}\right),\left|\left(k_{1}(\delta)\right)^{+}\right|=\left|k_{1}\left(\delta^{+}\right)\right|=$ $\left|k_{1}\left(2^{\delta}\right)\right|=\left|2^{k_{1}(\delta)}\right|=\mid\left\{f: f: P_{\delta}\left(\lambda_{\delta}\right) \rightarrow \delta^{+}\right.$is a function $\}|=|\left[\delta^{+}\right]^{\lambda_{\delta}} \mid=\lambda_{\delta}^{+}$.

Work until otherwise specified in $M^{* *}$. Consider the "term forcing" partial ordering $\mathbb{T}^{*}$ (see [8], Section 1.2.5, page 8) associated with $\dot{\mathbb{T}}^{0}$, i.e., $\tau \in \mathbb{T}^{*}$ iff $\tau$ is a term in the forcing language with respect to $\mathbb{P}_{\delta}^{0} * \dot{\mathbb{S}}^{0}$ and $\Vdash_{\mathbb{P}_{\delta}^{0} * \dot{\mathbb{S}}^{0}}$ " $\tau \in \dot{\mathbb{T}}^{0}$ ", ordered by $\tau \geq \sigma$ iff $\Vdash_{\mathbb{P}_{\delta}^{0} * \dot{\mathbb{S}}^{0}}$ " $\tau \geq \sigma$ ". Although $\mathbb{T}^{*}$ as defined is technically a proper class, it is possible to restrict the terms appearing in it to a sufficiently large set-sized collection, with the additional crucial property that any term $\tau$ forced to be in $\dot{T}^{0}$ is also forced to be equal to an element of $\mathbb{T}^{*}$. As we will show below, this can be done in such a way that $M^{* *} \vDash "\left|\mathbb{T}^{*}\right|=k_{1}(\delta)$ ".

Clearly, $\mathbb{T}^{*} \in M^{* *}$. Also, since $\Vdash_{\mathbb{P}_{\delta}^{0} * \dot{\mathbb{S}}^{0}}$ " $\mathbb{T}^{0}$ is $\prec \lambda_{\delta}^{+}$-strategically closed", it can easily be verified that $\mathbb{T}^{*}$ itself is $\prec \lambda_{\delta}^{+}$-strategically closed in $M^{* *}$ and, since $\left(M^{* *}\right)^{\lambda_{\delta}} \subseteq M^{* *}$, in $V$ as well.

Observe that $M^{* *} \vDash " k_{1}(\delta)$ is measurable and $\left|\mathbb{P}_{\delta}^{0} * \dot{\mathbb{S}}^{0}\right|<k_{1}(\delta)$ " and $\vdash_{\mathbb{P}_{\delta}^{0} * \dot{\mathbb{S}}^{0}}$ " $\dot{\mathbb{T}}^{0}$ is an Easton support iteration of length $k_{1}(\delta)$ and $\left|\dot{\mathbb{T}}^{0}\right|=k_{1}(\delta)$ ". 
We can thus let $\dot{f}$ be a term so that $\Vdash_{\mathbb{P}_{\delta}^{0} * \dot{S}^{0}}$ " $\dot{f}: k_{1}(\delta) \rightarrow \dot{\mathbb{T}}^{0}$ is a bijection". Since $M^{* *} \vDash "\left|\mathbb{P}_{\delta}^{0} * \dot{\mathbb{S}}^{0}\right|<k_{1}(\delta)$ ", for each $\alpha<k_{1}(\delta)$, let $S_{\alpha}=\left\{r_{\beta}^{\alpha}: \beta<\right.$ $\left.\eta^{\alpha}<k_{1}(\delta)\right\}$ be a maximal incompatible set of elements of $\mathbb{P}_{\delta}^{0} * \dot{\mathbb{S}}^{0}$ so that for some term $\tau_{\beta}^{\alpha}, r_{\beta}^{\alpha} \Vdash$ " $\tau_{\beta}^{\alpha}=\dot{f}(\alpha)$ ". Define $T_{\alpha}=\left\{\tau_{\beta}^{\alpha}: \beta<\eta^{\alpha}\right\}$ and $T=\bigcup_{\alpha<k_{1}(\delta)} T_{\alpha}$. Clearly, $|T|=k_{1}(\delta)$, so we can let $\left\langle\tau_{\alpha}: \alpha<k_{1}(\delta)\right\rangle$ enumerate the members of $T$. $\left\langle\tau_{\alpha}: \alpha<k_{1}(\delta)\right\rangle$ is so that if $\Vdash_{\mathbb{P}_{\delta}^{0} * \dot{\mathbb{S}}^{0}}$ " $\tau \in \dot{\mathbb{T}}^{0}$ ", then for some $\alpha<k_{1}(\delta), \Vdash_{\mathbb{P}_{\delta}^{0} * \dot{S}^{0}}$ " $\tau=\tau_{\alpha}$ ". Therefore, we can restrict the set of terms we choose so that we can assume that in $M^{* *},\left|\mathbb{T}^{*}\right|=k_{1}(\delta)$. Since $M^{* *} \vDash " 2^{k_{1}(\delta)}=\left(k_{1}(\delta)\right)^{+}=k_{1}\left(\delta^{+}\right)$", this means we can let $\left\langle D_{\alpha}: \alpha<\lambda_{\delta}^{+}\right\rangle$ enumerate in $V$ the dense open subsets of $\mathbb{T}^{*}$ found in $M^{* *}$, so that as before, for every dense open subset $D \subseteq \mathbb{T}^{*}$ present in $M^{* *}$ and some odd ordinal $\beta, D=D_{\beta}$, and argue as we did in Lemma 2.1 to construct in $V$ an $M^{* *}$-generic object $H^{*}$ over $\mathbb{T}^{*}$.

Note that since $N$ is given by an ultrapower of $M^{* *}$ via a normal ultrafilter $\mathcal{U}^{\prime \prime} \in M^{* *}$ over $\delta$, Fact 2 of Section 1.2.2 of [8] tells us that $k_{2}^{\prime \prime} H^{*}$ generates an $N$-generic object $H_{2}^{*}$ over $k_{2}\left(\mathbb{T}^{*}\right)$. By elementarity, $k_{2}\left(\mathbb{T}^{*}\right)$ is the term forcing in $N$ defined with respect to $k_{2}\left(k_{1}\left(\mathbb{P}_{\delta}^{0}\right)_{\delta+1}\right)=\mathbb{P}_{\delta}^{0} * \dot{\mathbb{Q}}^{0}$. Therefore, since $j^{*}\left(\mathbb{P}_{\delta}^{0}\right)=k_{2}\left(k_{1}\left(\mathbb{P}_{\delta}^{0}\right)\right)=\mathbb{P}_{\delta}^{0} * \dot{\mathbb{Q}}^{0} * \dot{\mathbb{R}}^{0}, H_{2}^{*}$ is $N$-generic over $k_{2}\left(\mathbb{T}^{*}\right)$, and $H_{0} * H_{1}^{0}$ is $k_{2}\left(\mathbb{P}_{\delta}^{0} * \dot{\mathbb{S}}^{0}\right)$-generic over $N$, Fact 1 of Section 1.2.5 of [8] tells us that for $H_{2}^{0}=\left\{i_{H_{0} * H_{1}^{0}}(\tau): \tau \in H_{2}^{*}\right\}, H_{2}^{0}$ is $N\left[H_{0}\right]\left[H_{1}^{0}\right]$-generic over $\mathbb{R}^{0}$.

Working in $V\left[H_{0}\right]\left[H_{1}\right]$, we build the generic objects $H_{1}^{1}$ and $H_{2}^{1}$. To construct $H_{1}^{1}$, we note that by the strategic closure properties of the partial orderings over which $H_{1}^{0}$ and $H_{2}^{0}$ are generic, $N\left[H_{0}\right]\left[H_{1}^{0}\right]\left[H_{2}^{0}\right]$ remains $\delta$ closed with respect to $V\left[H_{0}\right]\left[H_{1}^{0}\right]\left[H_{2}^{0}\right]=V\left[H_{0}\right]$. Therefore, since $\mathbb{P}_{\delta}^{1}$ is $\delta$-c.c., $N\left[H_{0}\right]\left[H_{1}^{0}\right]\left[H_{2}^{0}\right]\left[H_{1}\right]$ remains $\delta$ closed with respect to $V\left[H_{0}\right]\left[H_{1}^{0}\right]\left[H_{2}^{0}\right]\left[H_{1}\right]=$ $V\left[H_{0}\right]\left[H_{1}\right]$. This means we can construct $H_{1}^{1}$ in $V\left[H_{0}\right]\left[H_{1}\right]$ in the same way $H_{1}^{0}$ was constructed in $V\left[H_{0}\right]$.

To build $H_{2}^{1}$, we once again work in $M^{* *}$. Write $k_{1}\left(\mathbb{P}_{\delta}^{0} * \dot{\mathbb{P}}_{\delta}^{1}\right)$ as $\mathbb{P}_{\delta}^{0} * \dot{\mathbb{S}}^{0} *$ $\dot{\mathbb{T}}^{0} * \dot{\mathbb{P}}_{\delta}^{1} * \dot{\mathbb{T}}^{1}$, where $\dot{\mathbb{S}}^{0}$ and $\dot{\mathbb{T}}^{0}$ are as before, and $\dot{\mathbb{T}}^{1}$ is a term for the portion of $k_{1}\left(\mathbb{P}_{\delta}^{0} * \dot{\mathbb{P}}_{\delta}^{1}\right)$ defined in $M^{* *}$ between stages $\delta$ and $k_{1}(\delta)$. Since there are no measurable cardinals in $M^{* *}$ in the interval $\left(\delta, \lambda_{\delta}\right)$, the field of $\dot{T}^{1}$ is actually composed of ordinals in the interval $\left(\lambda_{\delta}, k_{1}(\delta)\right)$.

Let $\mathbb{T}^{* *}$ be the term forcing partial ordering associated with $\mathbb{T}^{1}$, i.e., $\tau \in \mathbb{T}^{* *}$ iff $\tau$ is a term in the forcing language with respect to $\mathbb{P}_{\delta}^{0} * \dot{\mathbb{S}}^{0} * \dot{\mathbb{T}}^{0} * \dot{\mathbb{P}}_{\delta}^{1}$ and $\vdash_{\mathbb{P}_{\delta}^{0} * \dot{\mathbb{S}}^{0} * \dot{\mathbb{T}}^{0} * \dot{\mathbb{P}}_{\delta}^{1}}$ “ $\tau \in \dot{\mathbb{T}} 1$ ", ordered by $\tau \geq \sigma$ iff $\vdash_{\mathbb{P}_{\delta}^{0} * \dot{\mathbb{S}}^{0} * \dot{\mathbb{T}}^{0} * \dot{\mathbb{P}}_{\delta}^{1}}$ " $\tau \geq \sigma$ ". A similar analysis to that given for the term forcing $\mathbb{T}^{*}$, using the observations made in the preceding paragraph, now allows us to construct in $V$ an $M^{* *}$-generic object $H^{* *}$ for $\mathbb{T}^{* *}$, infer that $k_{2}^{\prime \prime} H^{* *}$ generates an $N$-generic object $H_{2}^{* *}$ for the relevant term forcing partial ordering in $N$, and working 
in $V\left[H_{0}\right]\left[H_{1}\right]$, use $H_{2}^{* *}$ to create an $N\left[H_{0}\right]\left[H_{1}^{0}\right]\left[H_{2}^{0}\right]\left[H_{1}\right]\left[H_{1}^{1}\right]$-generic object $H_{2}^{1}$ over $\mathbb{T}^{1}$. Thus, in $V\left[H_{0}\right]\left[H_{1}\right], j^{*}: V \rightarrow N$ extends to $j^{*}: V\left[H_{0}\right]\left[H_{1}\right] \rightarrow$ $N\left[H_{0}\right]\left[H_{1}^{0}\right]\left[H_{2}^{0}\right]\left[H_{1}\right]\left[H_{1}^{1}\right]\left[H_{2}^{1}\right]$. This means $V^{\mathbb{P}_{\delta}^{0} * \dot{\mathbb{P}}_{\delta}^{1}}=V^{\mathbb{P}^{*}} \vDash " \delta$ is $\lambda_{\delta}$ strongly compact".

Claim 2. $V_{0}^{\mathbb{P}^{1}}=V^{\mathbb{P}} \vDash " \theta_{\delta}=\left(\theta_{\delta}\right)^{V_{0}}$.

Proof. To prove Claim 2, we assume inductively that $\delta$ is the least cardinal in $V_{0}$ for which this is not the case. As before, working in $V_{0}$, we can factor $\mathbb{P}^{1}$ as $\mathbb{P}^{\prime} * \dot{\mathbb{P}}^{\prime \prime}$, where $\left|\mathbb{P}^{\prime}\right|=\omega$ and $\Vdash_{\mathbb{P}^{\prime}}$ " $\dot{\mathbb{P}}^{\prime \prime}$ is $\aleph_{1}$-strategically closed". Thus, by the results of [9]-[11] if $V_{0}^{\mathbb{P}^{1}} \vDash$ " $\delta$ is $\gamma$ supercompact", $V_{0} \vDash$ " $\delta$ is $\gamma$ supercompact". This means that $V_{0}^{\mathbb{P}^{1}}=V^{\mathbb{P}} \vDash " \theta_{\delta} \leq\left(\theta_{\delta}\right)^{V_{0}}$ ", so by our assumptions on $\delta, V_{0}^{\mathbb{P}^{1}}=V^{\mathbb{P}} \vDash " \theta_{\delta}<\left(\theta_{\delta}\right)^{V_{0}}$ ".

Let $\gamma<\left(\theta_{\delta}\right)^{V_{0}}$ be either a regular cardinal or a singular (strong limit) cardinal of cofinality at least $\delta$. Choose an elementary embedding $i: V_{0} \rightarrow$ $M^{* * *}$ witnessing the $\gamma$ supercompactness of $\delta$ so that $V_{0} \vDash$ " $\delta$ is not $\gamma$ supercompact". By elementarity, since $V_{0} \vDash$ " $\delta$ is the least cardinal for which the first cardinal at which $\delta$ 's supercompactness fails decreases in size when forcing with $\mathbb{P}^{1}$ ", $M^{* * *} \vDash " i(\delta)>\delta$ is the least cardinal for which the first cardinal at which $i(\delta)$ 's supercompactness fails decreases in size when forcing with $i\left(\mathbb{P}^{1}\right)$ ". By GCH in $V_{0}$ and $M^{* * *}, M^{* * *} \vDash " \theta_{\delta}=\gamma$ ". Therefore, by the definition of $\mathbb{P}^{1}$ and the choice of $i, i\left(\mathbb{P}^{1}\right)=\mathbb{P}^{1} * \dot{\mathbb{Q}}^{\prime}$, where the least ordinal in the field of $\dot{\mathbb{Q}}^{\prime}$ is above $\left(\gamma^{+}\right)^{M^{* * *}}=\left(\gamma^{+}\right)^{V_{0}}$. Another application of GCH in $V_{0}$ now allows us once again to apply the argument given in Lemma 2.1 to show that for $G V_{0}$-generic over $\mathbb{P}^{1}$ and $G_{\delta}=G\left\lceil\mathbb{P}_{\delta}^{1}\right.$, in $V_{0}\left[G_{\delta}\right], i$ extends to $i: V_{0}\left[G_{\delta}\right] \rightarrow M^{* * *}\left[G_{\delta}\right]\left[G^{*}\right]$, where $G^{*}$ is an $M^{* * *}\left[G_{\delta}\right]$-generic object over $i\left(\mathbb{P}_{\delta}^{1}\right) / G_{\delta}$ constructed in $V_{0}\left[G_{\delta}\right]$. If we now write $G=G_{\delta} * G^{\delta}$, then since $G^{\delta}$ is $V_{0}\left[G_{\delta}\right]$-generic over a partial ordering which is $\left(\theta_{\delta}^{+5}\right)^{V_{0}}$-strategically closed and $\gamma<\left(\theta_{\delta}\right)^{V_{0}}<\left(\theta_{\delta}^{+5}\right)^{V_{0}}, V_{0}^{\mathbb{P}^{1}}=V^{\mathbb{P}} \vDash$ " $\delta$ is $\gamma$ supercompact". Since $\gamma$ can be any regular cardinal or singular strong limit cardinal of cofinality at least $\delta$ below $\left(\theta_{\delta}\right)^{V_{0}}$, and since $\delta$ 's supercompactness cannot first fail at the successor of a singular (strong limit) cardinal of cofinality below $\delta$ (this is because if $\eta>\delta$ is a singular strong limit cardinal of cofinality below $\delta$, then $\delta$ is $\eta$ supercompact iff $\delta$ is $\eta^{+}$supercompact), we have shown in contradiction to what we deduced above that $V_{0}^{\mathbb{P}^{1}}=V^{\mathbb{P}} \vDash " \theta_{\delta}=\left(\theta_{\delta}\right) V_{0}$ ".

Claim 3. Claims 1 and 2 allow us to finish as in Case 1.

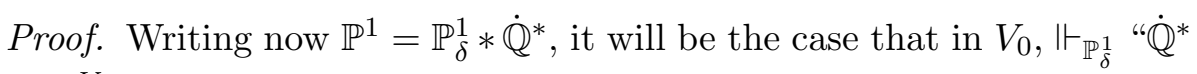
is $\left(\theta_{\delta}^{+5}\right)^{V_{0}}$-strategically closed and adds a non-reflecting stationary set of ordinals of cofinality $\omega$ to $\left(\theta_{\delta}^{+6}\right)^{V_{0}}$ ". By Claims 1 and 2 , we can therefore conclude as we did in Case 1 above that $V_{0}^{\mathbb{P}_{\delta}^{1} * \mathbb{Q}^{*}}=V_{0}^{\mathbb{P}^{1}}=V^{\mathbb{P}} \vDash " \delta$ is 
$<\theta_{\delta}$ supercompact, $\delta$ is $\theta_{\delta}^{+5}$ strongly compact, and $\delta$ is not $\theta_{\delta}^{+6}$ strongly compact".

The proof of Claim 3 completes the proof of Lemma 2.3.

Let now $V^{*}$ be $V^{\mathbb{P}}$ truncated at $\lambda$. Lemmas $2.1-2.3$ show that $V^{*}$ is our desired model. This completes the proof of Theorem 2.

We take this opportunity to make some concluding observations. First, we note that the proof of Theorem 2 remains valid if the cofinality of the ordinals in the non-reflecting stationary sets added is changed to any regular cardinal below the least $V$-measurable cardinal. Also, as we remarked in Section 1, and as the proof just given indicates, there is nothing special about +5 indicating the degree of level by level inequivalence between strong compactness and supercompactness below $\kappa$. There are two things critical to our proof, however, concerning this notion. In Section 1, we observed that it is possible for the degree of level by level inequivalence between strong compactness and supercompactness for a measurable cardinal $\delta<\kappa$ to be given by $\varphi(\delta)$, where $\varphi(\delta)$ defines an ordinal below $\lambda_{\delta}$, subject to the restriction that $\theta_{\delta}^{+\varphi(\delta)}$ cannot be a singular strong limit cardinal of cofinality below $\delta$. This is since if $\delta$ is $\gamma$ strongly compact where $\gamma$ is a singular strong limit cardinal of cofinality $\eta<\delta$, then $\delta$ in fact must be $\gamma^{+}$strongly compact. Also, because we do not yet know how to obtain a model in which every measurable cardinal manifests as much strong compactness as desired (see the discussion given after the proofs of Theorems 1 and 2 of [3]), we of necessity have to restrict the size of our universe in the proof just given. Thus, a question we pose to conclude Section 2 is whether it is possible to prove a version of Theorem 2 in a universe containing as many large cardinals as desired.

3. The proof of Theorem 3. Let $V \vDash " \mathrm{ZFC}+\mathfrak{K}$ is the class of supercompact cardinals". Without loss of generality, by first doing a preliminary forcing if necessary, we may also assume that $V$ is as in Theorem 1, i.e., that GCH and level by level equivalence between strong compactness and supercompactness hold in $V$. This allows us to define in $V$ our partial ordering $\mathbb{P}$ as the Easton support iteration which first adds a Cohen real and then, at any inaccessible cardinal $\delta$, forces with $\operatorname{Add}\left(\delta, \delta^{++}\right)$. Standard arguments in tandem with the results of [9]-[11] then show that cardinals and cofinalities are preserved when forcing with $\mathbb{P}$ and $V^{\mathbb{P}} \vDash$ "ZFC $+\mathfrak{K}$ is the class of supercompact cardinals $+2^{\delta}=\delta^{++}$if $\delta$ is inaccessible $+2^{\delta}=\delta^{+}$if $\delta$ is not inaccessible". Thus, the proof of Theorem 3 will be complete once we have established the following two lemmas. 
LEMMA 3.1. If $V \vDash " \kappa<\lambda$ are so that $\kappa$ is $\lambda$ supercompact and $\lambda$ is regular but not inaccessible", then $V^{\mathbb{P}} \vDash$ " $\kappa$ is $\lambda$ supercompact".

Proof. If $\kappa$ and $\lambda$ are as in the hypotheses of Lemma 3.1, then we consider the following two cases.

CAsE 1: $\lambda$ is not the successor of an inaccessible cardinal. Write $\mathbb{P}=$ $\mathbb{P}_{\lambda} * \dot{\mathbb{P}}^{\lambda}$, where the field of $\mathbb{P}_{\lambda}$ consists of ordinals below $\lambda$, and $\mathbb{P}^{\lambda}$ consists of the rest of $\mathbb{P}$. By the choice of $\lambda,\left|\mathbb{P}_{\lambda}\right| \leq \lambda$. Hence, by the definition of $\mathbb{P}_{\lambda}$, if $j: V \rightarrow M$ is an embedding witnessing the $\lambda$ supercompactness of $\kappa, \mathbb{P}_{\lambda}$ is an initial segment of $j\left(\mathbb{P}_{\lambda}\right)$. Therefore, the standard reverse Easton arguments

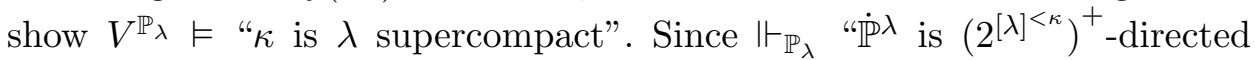
closed", $V^{\mathbb{P}_{\lambda} * \dot{\mathbb{P}}^{\lambda}}=V^{\mathbb{P}} \vDash " \kappa$ is $\lambda$ supercompact".

CASE 2: $\lambda$ is the successor of an inaccessible cardinal. Once again, write $\mathbb{P}=\mathbb{P}_{\lambda} * \dot{\mathbb{P}}^{\lambda}$, where the field of $\mathbb{P}_{\lambda}$ consists of ordinals below $\lambda$, and $\mathbb{P}^{\lambda}$ is the rest of $\mathbb{P}$, i.e., the field of $\mathbb{P}^{\lambda}$ consists of ordinals above $\lambda$. In this instance, it is not the case that $\left|\mathbb{P}_{\lambda}\right| \leq \lambda$, since for the $\delta$ so that $\lambda=\delta^{+}$, $\left|\mathbb{P}_{\lambda}\right|=\delta^{++}=\lambda^{+}>\lambda$. However, the arguments given on pages 119-120 of [6] or pages 555-556 of [1] (which are originally due to Magidor and are also found earlier in [12], [13], and [17]) will yield that $V^{\mathbb{P}_{\lambda}} \vDash " \kappa$ is $\lambda=\delta^{+}$ supercompact". For the convenience of the readers, we give these arguments below.

Write $\mathbb{P}_{\lambda}=\mathbb{Q}_{0} * \dot{\mathbb{Q}}_{1} * \operatorname{Add}\left(\delta, \delta^{++}\right)$, where the field of $\mathbb{Q}_{0}$ consists of ordinals below $\kappa$, and the field of $\dot{\mathbb{Q}}^{1}$ is composed of all remaining ordinals in the interval $[\kappa, \lambda)$. Let $G$ be $V$-generic over $\mathbb{P}_{\lambda}$, with $G_{0} * G_{1} * G_{2}$ the corresponding factorization of $G$. Fix an elementary embedding $j: V \rightarrow$ $M$ witnessing the $\lambda=\delta^{+}$supercompactness of $\kappa$ which is generated by a supercompact ultrafilter $\mathcal{U}$ over $P_{\kappa}(\lambda)$. We then have $j\left(\mathbb{P}_{\lambda}\right)=\mathbb{Q}_{0} * \dot{\mathbb{Q}}_{1} *$ $\operatorname{Add}\left(\delta, \delta^{++}\right) * \dot{\mathbb{R}}_{0} * \dot{\mathbb{R}}_{1}$, where $\dot{\mathbb{R}}_{1}$ is a term for $\operatorname{Add}\left(j(\delta), j\left(\delta^{++}\right)\right)$as computed

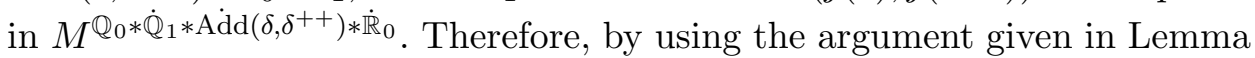
2.1 , since $M\left[G_{0}\right]\left[G_{1}\right]\left[G_{2}\right]$ remains $\lambda$ closed with respect to $V\left[G_{0}\right]\left[G_{1}\right]\left[G_{2}\right]$ and $V \vDash \mathrm{GCH}$, it is possible working in $V\left[G_{0}\right]\left[G_{1}\right]\left[G_{2}\right]$ to construct an $M\left[G_{0}\right]\left[G_{1}\right]\left[G_{2}\right]$-generic object $G_{3}$ over $\mathbb{R}_{0}$ and extend $j$ to $j: V\left[G_{0}\right]\left[G_{1}\right] \rightarrow$ $M\left[G_{0}\right]\left[G_{1}\right]\left[G_{2}\right]\left[G_{3}\right]$. It is then the case that $M\left[G_{0}\right]\left[G_{1}\right]\left[G_{2}\right]\left[G_{3}\right]$ remains $\lambda$ closed with respect to $V\left[G_{0}\right]\left[G_{1}\right]\left[G_{2}\right]$.

For $\alpha \in\left(\delta, \delta^{++}\right)$and $p \in \operatorname{Add}\left(\delta, \delta^{++}\right)$, let $p\lceil\alpha=\{\langle\langle\varrho, \sigma\rangle, \eta\rangle \in p: \sigma<$ $\alpha\}$ and $G_{2}\left\lceil\alpha=\left\{p\left\lceil\alpha: p \in G_{2}\right\}\right.\right.$. Clearly, $V\left[G_{0}\right]\left[G_{1}\right]\left[G_{2}\right] \vDash " \mid G_{2}\left\lceil\alpha \mid \leq \delta^{+}\right.$ for all $\alpha \in\left(\delta, \delta^{++}\right)$". Thus, since $\operatorname{Add}\left(j(\delta), j\left(\delta^{++}\right)\right)^{M\left[G_{0}\right]\left[G_{1}\right]\left[G_{2}\right]\left[G_{3}\right]}$ is $j(\delta)$ directed closed and $j(\delta)>\delta^{++}, q_{\alpha}=\bigcup\left\{j(p): p \in G_{2}\lceil\alpha\}\right.$ is well-defined and is an element of $\operatorname{Add}\left(j(\delta), j\left(\delta^{++}\right)\right)^{M\left[G_{0}\right]\left[G_{1}\right]\left[G_{2}\right]\left[G_{3}\right]}$. Further, if $\langle\varrho, \sigma\rangle \in$ $\operatorname{dom}\left(q_{\alpha}\right)-\operatorname{dom}\left(\bigcup_{\beta<\alpha} q_{\beta}\right) \quad\left(\bigcup_{\beta<\alpha} q_{\beta}\right.$ is well-defined by closure), then $\sigma \in$ $\left[\bigcup_{\beta<\alpha} j(\beta), j(\alpha)\right)$. To see this, assume to the contrary that $\sigma<\bigcup_{\beta<\alpha} j(\beta)$. 
Let $\beta$ be minimal so that $\sigma<j(\beta)$. It must thus be the case that for some $p \in G_{2} \mid \alpha,\langle\varrho, \sigma\rangle \in \operatorname{dom}(j(p))$. Since by elementarity and the definitions of $G_{2} \uparrow \beta$ and $G_{2}\left\lceil\alpha\right.$, for $p\left\lceil\beta=q \in G_{2}\lceil\beta, j(q)=j(p)\lceil j(\beta)=j(p\lceil\beta)\right.$, it must be the case that $\langle\varrho, \sigma\rangle \in \operatorname{dom}(j(q))$. This means $\langle\varrho, \sigma\rangle \in \operatorname{dom}\left(q_{\beta}\right)$, a contradiction.

Since $M\left[G_{0}\right]\left[G_{1}\right]\left[G_{2}\right]\left[G_{3}\right] \vDash$ "GCH holds for all cardinals $\geq j(\delta)$ ", $M\left[G_{0}\right]\left[G_{1}\right]\left[G_{2}\right]\left[G_{3}\right] \vDash " \operatorname{Add}\left(j(\delta), j\left(\delta^{++}\right)\right)$is $j\left(\delta^{+}\right)$-c.c. and has $j\left(\delta^{++}\right)$many maximal antichains". This means that if $\mathcal{A} \in M\left[G_{0}\right]\left[G_{1}\right]\left[G_{2}\right]\left[G_{3}\right]$ is a maximal antichain of $\operatorname{Add}\left(j(\delta), j\left(\delta^{++}\right)\right)$, then $\mathcal{A} \subseteq \operatorname{Add}(j(\delta), \beta)$ for some $\beta \in\left(j(\delta), j\left(\delta^{++}\right)\right)$. Thus, since GCH in $V$ and the fact $j$ is generated by a supercompact ultrafilter over $P_{\kappa}\left(\delta^{+}\right)$imply that $V \vDash "\left|j\left(\delta^{++}\right)\right|=\delta^{++}$", we can let $\left\langle\mathcal{A}_{\alpha}: \alpha \in\left(\delta, \delta^{++}\right)\right\rangle \in V\left[G_{0}\right]\left[G_{1}\right]\left[G_{2}\right]$ be an enumeration of all the maximal antichains of $\operatorname{Add}\left(j(\delta), j\left(\delta^{++}\right)\right)$present in $M\left[G_{0}\right]\left[G_{1}\right]\left[G_{2}\right]\left[G_{3}\right]$.

Working in $V\left[G_{0}\right]\left[G_{1}\right]\left[G_{2}\right]$, we define now an increasing sequence $\left\langle r_{\alpha}\right.$ : $\left.\alpha \in\left(\delta, \delta^{++}\right)\right\rangle$of elements of $\operatorname{Add}\left(j(\delta), j\left(\delta^{++}\right)\right)$so that $\forall \alpha \in\left(\delta, \delta^{++}\right)\left[r_{\alpha} \geq q_{\alpha}\right.$ and $\left.r_{\alpha} \in \operatorname{Add}(j(\delta), j(\alpha))\right]$ and so that $\forall \mathcal{A} \in\left\langle\mathcal{A}_{\alpha}: \alpha \in\left(\delta, \delta^{++}\right)\right\rangle \exists \beta \in$ $\left(\delta, \delta^{++}\right) \exists r \in \mathcal{A}\left[r_{\beta} \geq r\right]$. Assuming we have such a sequence, $G_{4}=\{p \in$ $\operatorname{Add}\left(j(\delta), j\left(\delta^{++}\right)\right): \exists r \in\left\langle r_{\alpha}: \alpha \in\left(\delta, \delta^{++}\right)\right\rangle[r \geq p]$ is an $M\left[G_{0}\right]\left[G_{1}\right]\left[G_{2}\right]\left[G_{3}\right]-$ generic object over $\left.\operatorname{Add}\left(j(\delta), j\left(\delta^{++}\right)\right)\right\}$. To define $\left\langle r_{\alpha}: \alpha \in\left(\delta, \delta^{++}\right)\right\rangle$, if $\alpha$ is a limit, we let $r_{\alpha}=\bigcup_{\beta \in(\delta, \alpha)} r_{\beta}$. By the facts $\left\langle r_{\beta}: \beta \in(\delta, \alpha)\right\rangle$ is (strictly) increasing and $M\left[G_{0}\right]\left[G_{1}\right]\left[G_{2}\right]\left[G_{3}\right]$ is $\delta^{+}$closed with respect to $V\left[G_{0}\right]\left[G_{1}\right]\left[G_{2}\right]$, this definition is valid. Assuming now $r_{\alpha}$ has been defined and we wish to define $r_{\alpha+1}$, let $\left\langle\mathcal{B}_{\beta}: \beta<\eta \leq \delta^{+}\right\rangle$be the subsequence of $\left\langle\mathcal{A}_{\beta}: \beta \leq \alpha+1\right\rangle$ containing each antichain $\mathcal{A}$ so that $\mathcal{A} \subseteq \operatorname{Add}(j(\delta), j(\alpha+1))$. Since $q_{\alpha}, r_{\alpha} \in$ $\operatorname{Add}(j(\delta), j(\alpha)), q_{\alpha+1} \in \operatorname{Add}(j(\delta), j(\alpha+1))$, and $j(\alpha)<j(\alpha+1)$, the condition $r_{\alpha+1}^{\prime}=r_{\alpha} \cup q_{\alpha+1}$ is well-defined, since by our earlier observations, any new elements of $\operatorname{dom}\left(q_{\alpha+1}\right)$ will not be present in either $\operatorname{dom}\left(q_{\alpha}\right)$ or $\operatorname{dom}\left(r_{\alpha}\right)$. We can thus, using the fact that $M\left[G_{0}\right]\left[G_{1}\right]\left[G_{2}\right]\left[G_{3}\right]$ is closed under $\delta^{+}$sequences with respect to $V\left[G_{0}\right]\left[G_{1}\right]\left[G_{2}\right]$, define by induction an increasing sequence $\left\langle s_{\beta}: \beta<\eta\right\rangle$ so that $s_{0} \geq r_{\alpha+1}^{\prime}, s_{\varrho}=\bigcup_{\beta<\varrho} s_{\beta}$ if $\varrho$ is a limit ordinal, and $s_{\beta+1} \geq s_{\beta}$ is so that $s_{\beta+1}$ extends some element of $\mathcal{B}_{\beta}$. The just mentioned closure fact implies $r_{\alpha+1}=\bigcup_{\beta<\eta} s_{\beta}$ is a well-defined condition.

In order to show that $G_{4}$ is $M\left[G_{0}\right]\left[G_{1}\right]\left[G_{2}\right]\left[G_{3}\right]$-generic over $\operatorname{Add}\left(j(\delta), j\left(\delta^{++}\right)\right)$, we must show that $\forall \mathcal{A} \in\left\langle\mathcal{A}_{\alpha}: \alpha \in\left(\delta, \delta^{++}\right)\right\rangle \exists \beta \in$ $\left(\delta, \delta^{++}\right) \exists r \in \mathcal{A}\left[r_{\beta} \geq r\right]$. To do this, we first note that $\left\langle j(\alpha): \alpha<\delta^{++}\right\rangle$is unbounded in $j\left(\delta^{++}\right)$. To see this, if $\beta<j\left(\delta^{++}\right)$is an ordinal, then for some $f: P_{\kappa}\left(\delta^{+}\right) \rightarrow M$ representing $\beta$, we can assume that for $p \in P_{\kappa}\left(\delta^{+}\right), f(p)<$ $\delta^{++}$. Thus, by the regularity of $\delta^{++}$in $V, \beta_{0}=\bigcup_{p \in P_{\kappa}\left(\delta^{+}\right)} f(p)<\delta^{++}$, and $j\left(\beta_{0}\right)>\beta$. This means by our earlier remarks that if $\mathcal{A} \in\left\langle\mathcal{A}_{\alpha}: \alpha<\delta^{++}\right\rangle$, $\mathcal{A}=\mathcal{A}_{\varrho}$, then we can let $\beta \in\left(\delta, \delta^{++}\right)$be so that $\mathcal{A} \subseteq \operatorname{Add}(j(\delta), j(\beta))$. By construction, for $\eta>\max (\beta, \varrho)$, there is some $r \in \mathcal{A}$ so that $r_{\eta} \geq r$. 
And, as any $p \in \operatorname{Add}\left(\delta, \delta^{++}\right)$is so that for some $\alpha \in\left(\delta, \delta^{++}\right), p=p\left\lceil\alpha, G_{4}\right.$ is so that if $p \in G_{2}, j(p) \in G_{4}$. Thus, working in $V\left[G_{0}\right]\left[G_{1}\right]\left[G_{2}\right]$, we have shown that $j$ extends to $j: V\left[G_{0}\right]\left[G_{1}\right]\left[G_{2}\right] \rightarrow M\left[G_{0}\right]\left[G_{1}\right]\left[G_{2}\right]\left[G_{3}\right]\left[G_{4}\right]$, i.e., $V\left[G_{0}\right]\left[G_{1}\right]\left[G_{2}\right] \vDash " \kappa$ is $\lambda=\delta^{+}$supercompact". Since as in Case $1, \vdash_{\mathbb{P}_{\lambda}}$ " $\dot{\mathbb{P}}^{\lambda}$ is $\left(2^{[\lambda]^{<\kappa}}\right)^{+}$-directed closed", $V^{\mathbb{P}_{\lambda} * \dot{\mathbb{P}}^{\lambda}}=V^{\mathbb{P}} \vDash " \kappa$ is $\lambda$ supercompact". This completes the proof of Case 2 and Lemma 3.1.

We observe that the proof of Lemma 3.1 breaks down if $\lambda$ is inaccessible. This is since the inner model $M$ will not have enough closure to allow either of the proofs given in Cases 1 and 2 above to remain valid.

LEMMA 3.2. $V^{\mathbb{P}} \vDash$ "For every pair of regular cardinals $\kappa<\lambda, \kappa$ is $\lambda$ strongly compact iff $\kappa$ is $\lambda$ supercompact, except possibly if $\kappa$ is a measurable limit of cardinals $\delta$ which are $\lambda$ supercompact, or $\lambda$ is inaccessible".

Proof. Suppose $V^{\mathbb{P}} \vDash " \kappa<\lambda$ are regular, $\lambda$ is not inaccessible, $\kappa$ is $\lambda$ strongly compact, and $\kappa$ is not a measurable limit of cardinals $\delta$ which are $\lambda$ supercompact". Since forcing with $\mathbb{P}$ preserves all cardinals and cofinalities, $V \vDash$ " $\lambda$ is regular but is not inaccessible". Therefore, by Lemma 3.1, any cardinal $\delta$ so that $V \vDash$ " $\delta$ is $\lambda$ supercompact" remains $\lambda$ supercompact in $V^{\mathbb{P}}$. This means $V \vDash " \kappa<\lambda$ are regular, $\lambda$ is not inaccessible, and $\kappa$ is not a measurable limit of cardinals $\delta$ which are $\lambda$ supercompact", and by the results of [9]-[11], $V \vDash$ " $\kappa$ is $\lambda$ strongly compact". Hence, by level by level equivalence in $V, V \vDash$ " $\kappa$ is $\lambda$ supercompact", so another application of Lemma 3.1 implies that $V^{\mathbb{P}} \vDash$ " $\kappa$ is $\lambda$ supercompact". This completes the proof of Lemma 3.2.

\section{Lemmas 3.1 and 3.2 complete the proof of Theorem 3 .}

We note that any cardinal $\kappa$ in $V^{\mathbb{P}}$ which is a measurable limit of cardinals $\delta$ which are $\lambda$ strongly compact where $\lambda>\kappa$ is regular but not inaccessible must be in $V^{\mathbb{P}}$ a measurable limit of cardinals $\delta$ which are $\lambda$ supercompact. This is since the results of [9]-[11], which tell us that there are no new instances of measurability, strong compactness, or supercompactness in $V^{\mathbb{P}}$, imply that $\kappa$ must be in $V$ a measurable limit of cardinals $\delta$ which are $\lambda$ strongly compact. $\kappa$ can then be written in $V$ as a measurable limit of cardinals $\delta$ which are $\lambda$ strongly compact where each such $\delta$ is not itself a measurable limit of cardinals $\gamma$ which are $\lambda$ strongly compact. By level by level equivalence between strong compactness and supercompactness in $V$, each such cardinal $\delta$ must be $\lambda$ supercompact in $V$. Lemma 3.1 then implies that each of these cardinals remains $\lambda$ supercompact in $V^{\mathbb{P}}$.

In conclusion to this paper, we mention that Theorem 3 raises a number of open questions. Some of these are as follows:

1. In the model for Theorem 3, is there precise level by level equivalence between strong compactness and supercompactness? If not, is there any 
model for precise level by level equivalence between strong compactness and supercompactness in which $2^{\delta}=\delta^{++}$for every inaccessible cardinal $\delta$ ?

2. Is it possible to get a model witnessing the conclusions of Theorem 3 in which there are different kinds of failures of $\mathrm{GCH}$ at the inaccessible cardinals, e.g., in which $2^{\delta}=\delta^{+++}$for every inaccessible cardinal $\delta$, or $2^{\delta}=\delta^{++}$at every regular cardinal?

3. In general, what sorts of failures of $\mathrm{GCH}$ are possible in a model for precise level by level equivalence between strong compactness and supercompactness?

\section{References}

[1] A. Apter, Forcing the least measurable to violate GCH, Math. Logic Quart. 45 (1999), 551-560.

[2] - Some structural results concerning supercompact cardinals, J. Symbolic Logic 66 (2001), 1919-1927.

[3] -, Strong compactness, measurability, and the class of supercompact cardinals, Fund. Math. 167 (2001), 65-78.

[4] A. Apter and J. Cummings, Identity crises and strong compactness, J. Symbolic Logic 65 (2000), 1895-1910.

[5] -, 一, Identity crises and strong compactness II: strong cardinals, Arch. Math. Logic 40 (2001), 25-38.

[6] A. Apter and S. Shelah, On the strong equality between supercompactness and strong compactness, Trans. Amer. Math. Soc. 349 (1997), 103-128.

[7] J. Burgess, Forcing, in: J. Barwise (ed.), Handbook of Mathematical Logic, NorthHolland, Amsterdam, 1977, 403-452.

[8] J. Cummings, A model in which GCH holds at successors but fails at limits, Trans. Amer. Math. Soc. 329 (1992), 1-39.

[9] J. D. Hamkins, Destruction or preservation as you like it, Ann. Pure Appl. Logic 91 (1998), 191-229.

[10] —, Gap forcing, Israel J. Math. 125 (2001), 237-252.

[11] —, Gap forcing: Generalizing the Lévy-Solovay theorem, Bull. Symbolic Logic 5 (1999), 264-272.

[12] T. Jech, M. Magidor, W. Mitchell and K. Prikry, Precipitous ideals, J. Symbolic Logic 45 (1980), 1-8.

[13] T. Jech and W. H. Woodin, Saturation of the closed unbounded filter on the set of regular cardinals, Trans. Amer. Math. Soc. 292 (1985), 345-356.

[14] A. Kanamori, The Higher Infinite, Springer, Berlin, 1994.

[15] Y. Kimchi and M. Magidor, The independence between the concepts of compactness and supercompactness, circulated manuscript.

[16] A. Lévy and R. Solovay, Measurable cardinals and the continuum hypothesis, Israel J. Math. 5 (1967), 234-248.

[17] M. Magidor, On the existence of nonregular ultrafilters and the cardinality of ultrapowers, Trans. Amer. Math. Soc. 249 (1979), 97-111.

[18] T. Menas, On strong compactness and supercompactness, Ann. Math. Logic 7 (1974), $327-359$. 
[19] R. Solovay, W. Reinhardt and A. Kanamori, Strong axioms of infinity and elementary embeddings, ibid. 13 (1978), 73-116.

Department of Mathematics

Baruch College of CUNY

New York, NY 10010, U.S.A.

E-mail: awabb@cunyvm.cuny.edu

http://math.baruch.cuny.edu/ apter

Received 21 February 2001;

in revised form 26 August 2001 